\title{
Pómez Bosque de Tlalpan, producto de una erupción de gran magnitud en el margen suroeste de la cuenca de México
}

\author{
José Luis Arce ${ }^{1, *}$, Diana Cruz-Fuentes ${ }^{2}$, Angel Ramírez-Luna ${ }^{3}$, \\ Iván Andrés Herrera-Huerta ${ }^{2}$ y Patricia Girón-García ${ }^{1}$
}

${ }^{1}$ Instituto de Geología, Universidad Nacional Autónoma de México, Cd. Universitaria, Coyoacán, C.P. 04510, Ciudad de México, México.

${ }^{2}$ Escuela Superior de Ingeniería y Arquitectura (ESIA), Ticomán, Instituto Politécnico Nacional, C.P. 04510, Ciudad de México, México.

${ }^{3}$ Instituto de Geofísica, Universidad Nacional Autónoma de México, Cd. Universitaria, Coyoacán, C.P. 04510, Ciudad de México, México.

*jlarce@geologia.unam.mx

\section{RESUMEN}

El presente trabajo reporta por primera vez la descripción de un depósito de pómez de caída de color blanco que aflora en la parte sur de la cuenca de México, denominado aquí de manera informal como pómez Bosque de Tlalpan (PBT). El afloramiento más representativo se encuentra en el Bosque de Tlalpan, Delegación Tlalpan, de donde toma su nombre. En este sitio el depósito alcanza $3 \mathrm{~m}$ de espesor, es masivo, la pómez representa el $80-90 \%$ en volumen, con un predominio de tamaño del lapilli grueso y algunos bloques. La PBT es de composición dacítica (64.9-66 \% en peso de $\mathrm{SiO}_{2}$, en base anhidra) con afinidad calcialcalina, con una mineralogía de plagioclasa $>$ anfíbol $>$ ortopiroxeno > biotita, en menor proporción cuarzo, óxidos de Fe-Ti y circón como mineral accesorio, inmersos en una matriz vesicular y vítrea. De acuerdo con el análisis preliminar de afloramientos, el espesor y tamaño de los constituyentes del depósito disminuye hacia el $\mathrm{NE}$, por lo tanto es posible que la fuente de emisión se encuentre hacia el SW del Bosque de Tlalpan. En esta dirección se encuentra el volcán Ajusco (a $\sim 11 \mathrm{~km}$ de Bosque de Tlalpan) y el volcán San Miguel, ambos pertenecientes a la Sierra de Las Cruces y posiblemente uno de ellos fue el foco de emisión. La edad del depósito de la PBT fue estimada entre $25,730 \pm 130$ y $37,450 \pm 330$ años A.P. mediante el fechamiento por el método de radiocarbono de paleosuelos que subyacen al depósito, la cual ubica el depósito en el Pleistoceno Tardío. Adicionalmente un fechamiento por termoluminiscencia arrojó una edad de $30,300 \pm 5,000$ años, similar a las edades de radiocarbono, por lo que se puede asegurar que este depósito representa el producto más joven de la Sierra de Las Cruces. A pesar de contar con solo siete afloramientos hasta el momento, se plantea que este depósito fue producido por una erupción de tipo pliniano, ya que los afloramientos descritos son masivos, presentan espesores de alrededor de tres metros y dimensiones de la pómez de lapilli grueso hasta bloques, así como líticos de $3.5 \mathrm{~cm}$ de diámetro a $11 \mathrm{~km}$ de la posible fuente de emisión, característico para este tipo de depósitos. Se descarta que la fuente de emisión sea alguno de los volcanes monogenéticos del campo volcánico Chichinautzin dado que este tipo de volcanes no producen erupciones plinianas y son de composición relativamente básica.

Palabras clave: erupción pliniana; pómez Bosque de Tlalpan; Pleistoceno Tardío; Sierra de Las Cruces; cuenca de México; México.

\begin{abstract}
In this contribution we describe a white pumice fall deposit, informally named pómez Bosque de Tlalpan (PBT), found in several outcrops in the southern Mexico basin. The most representative sequence occurs at the Bosque de Tlalpan park, Delegación Tlalpan, in Mexico City. In this site the deposit is $3 \mathrm{~m}$ thick, massive, and contains $80-90 \mathrm{vol}$. \% of pumice clasts, predominantly of coarse lapilli-sized and some block-sized fragments. The PBT has a dacitic composition (64.9-66 wt. \% $\mathrm{SiO}_{2}$, on anhydrous basis) of calc-alkaline affinity, and a mineral assemblage represented by plagioclase $>$ amphibole $>$ orthopyroxene $>$ biotite, $\pm F e-T i$ oxides, with quartz in lesser proportions, and zircon as accesory mineral, in a vesicular and glassy matrix. According to data collected from seven outcrops, thickness and clast size of the deposit decrease towards the NE and therefore the vent source should be located to the SW of Bosque de Tlalpan. Possible sources are the volcanoes Ajusco and San Miguel, which are part of the Sierra de Las Cruces volcanic range, and are located in this direction at $\sim 11 \mathrm{~km}$ from Bosque de Tlalpan. The age of the PBT deposit was determined by radiocarbon dating of underlying paleosoils that yielded 25,730 \pm 130 to 37,450 \pm 330 yrs B.P. (Late Pleistocene). Additionally, thermoluminscence dating of one sample yielded an age of 30,300 $\pm 5,000$ years, similar to the radiocarbon ages. Hence, the $P B T$ represents the youngest reported deposit from the Sierra de Las Cruces volcanic range. Although only seven outcrops were described in this work, we interpret the PBT deposit as produced by a plinian-type eruption, based on its massive structure, its ca. $3 \mathrm{~m}$ thickness, and the presence of pumice clasts in coarse lapilli to block sizes, as well as $3.5 \mathrm{~cm}$ sized lithics, at $11 \mathrm{~km}$ from the possible vent source, characteristic of this kind of deposits. We discard the monogenetic volcanoes from
\end{abstract}


the Chichinautzin volcanic field as the vent source of the PBT deposit, because this kind of volcanoes is relatively mafic in composition and do not produce plinian-type deposits.

Key words: plinian eruption; Bosque de Tlalpan pumice; Late Pleistocene; Sierra de Las Cruces; basin of México; Mexico.

\section{INTRODUCCIÓN}

La cuenca de México es una depresión volcanotectónica que se localiza dentro del Cinturón Volcánico Transmexicano (Figura 1a). Esta depresión está rodeada por sierras volcánicas: al oeste se encuentra la Sierra de Las Cruces en dirección casi norte-sur (Figura 1b), con edades que varían de 3.7 Ma al norte (Mora-Alvarez et al., 1991; Aguirre-Díaz et al., 2006; Osete et al., 2000; Mejia et al., 2005) a $0.9 \mathrm{Ma}$ al sur (Arce et al., 2008); al oriente se encuentra la Sierra Nevada conformada por los estratovolcanes Tláloc, Telapón, Iztaccíhuatl y Popocatépetl (Figura 1b), con edades variables de 1.8 a $1.2 \mathrm{Ma}$ en el norte, hasta el reciente en el sur (Cadoux et al., 2011; Macias et al., 2012; Sosa-Ceballos et al., 2015); y al sur de la cuenca se encuentra el campo volcánico Chichinautzin (Figura 1b), constituido por más de 200 volcanes monogenéticos y derrames de lava fisurales (Bloomfield, 1975; Martin del Pozzo, 1982; Márquez et al., 1999), con edades desde 1.2 Ma (Arce et al., 2013a) y tan jóvenes como 1,600 años antes del presente (A.P.) (Siebe, 2000). Este vulcanismo monogenético fue el responsable de haber bloqueado el drenaje hacia el sur de la cuenca de México (Mooser, 1963; Arce et al., 2013b).

La estratigrafía del Pleistoceno de la cuenca de México ha sido construida a partir de descripciones de campo (Vázquez-Sánchez y Jaimes-Palomera, 1989; Arce et al., 2008, 2015) pero también a partir de pozos someros que se han llevado a cabo en los remanentes del

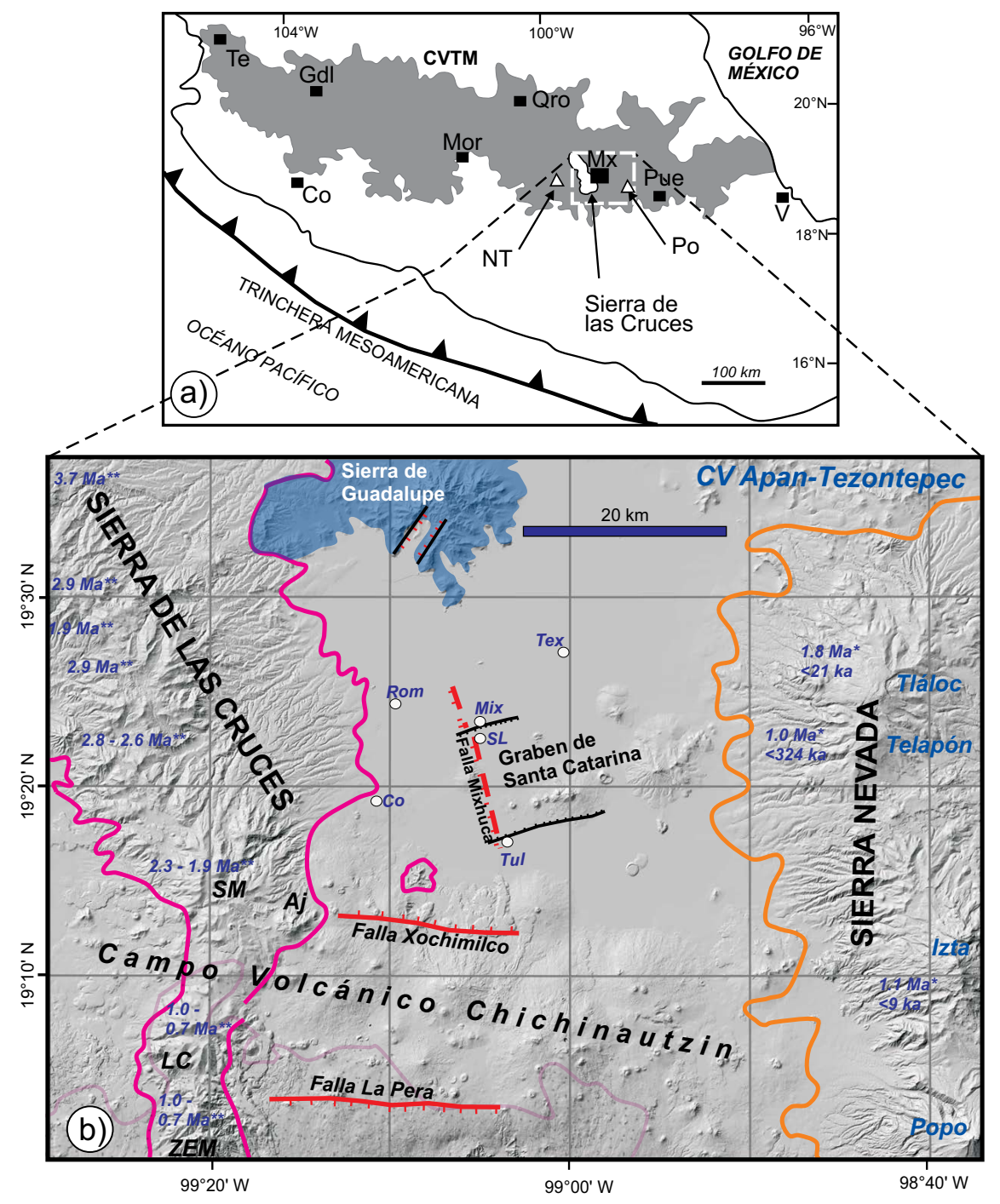

Figura 1. a) Mapa del Cinturón Volcánico Transmexicano (CVTM), mostrando la ubicación de la cuenca de México. El recuadro en el CVTM representa el área mostrada de b). Las estructuras volcánicas en a) son: Po, Popocatépetl y NT, Nevado de Toluca; mientras que las ciudades son: Co, Colima; Gdl, Guadalajara; Mor, Morelia; Mx, Ciudad de México; Pue, Puebla; Qro, Querétaro; Te, Tepic y V, Veracruz. b) Modelo digital de elevación de la parte sur de la cuenca de México en donde se aprecian las sierras volcánicas principales y demás elementos tectónicos. Estructuras volcánicas de la Sierra de Las Cruces: ZEM, volcán Zempoala; LC, caldera La Catedral; Aj, volcán Ajusco; SM, volcán San Miguel. Además se ubican los pozos profundos perforados por la SHCP en 1960 (Tex, Texcoco), por PEMEX, en los años 80's (Co, Copilco; Mix, Mixhuca; Rom, Roma; Tul, Tulyehualco) y por el SACMEX en el 2012 (pozo San Lorenzo Tezonco) (SL). Las edades fueron compiladas por Arce et al. (2015) tomando como referencia a varios autores. 
lago de Chalco (Lozano-García y Ortega-Guerrero, 1998; Caballero y Ortega-Guerrero, 1998; Brown et al., 2012). Dentro de estos trabajos se han reconocido depósitos lacustres y volcánicos, e incluso depósitos producidos por erupciones de gran magnitud de tipo pliniano provenientes de los volcanes Popocatépetl (Pómez Tutti Frutti de $\sim 14,000$ años A.P. (edad no calibrada); Siebe et al., 1995), localizado a $70 \mathrm{~km}$ al SE de la Ciudad de México y Nevado de Toluca (Pómez Toluca Superior de 10,500 años A.P. (edad no calibrada); Bloomfield et al., 1977; Arce et al., 2003), ubicado a $80 \mathrm{~km}$ al SW de la Ciudad de México.

En el presente trabajo se reporta el estudio de un depósito de pómez de caída, denominado de manera informal como pómez Bosque de Tlalpan (PBT), debido a que uno de los afloramientos más representativos se ubica en el parque del mismo nombre, localizado al sur de la cuenca de México (Figura 2), en la Delegación Tlalpan de la Ciudad de México. Con base en la descripción de afloramientos en campo, así como en obras civiles dentro de la mancha urbana, combinado con análisis químico de roca total, así como fechamientos por radiocarbono de paleosuelos y por el método de termoluminiscencia, se discute la fuente de emisión, el tipo de erupción y la edad del depósito PBT.

\section{METODOLOGÍA}

La metodología que se empleó para este estudio consistió en llevar a cabo descripciones de afloramientos tanto superficiales como en obras civiles dentro de la mancha urbana. Para esto se realizaron descripciones de los distintos horizontes de cada afloramiento, considerando características como color, estructura, espesor de cada depósito, tipos, dimensiones y abundancia de los constituyentes, variaciones laterales, entre otros. Además se realizó un muestreo de tres paleosuelos en tres distintos afloramientos para llevar a cabo fechamientos por medio de radiocarbono con la técnica de espectrometría de masas con aceleradores (AMS, Accelerator Mass Spectrometry), así como un fechamiento del depósito de pómez por termoluminiscencia en el afloramiento PBT-01 (Figura 2).

\section{Química de roca total}

De todos los afloramientos de la PBT (Tabla 1) se seleccionaron 11 muestras de pómez, las cuales fueron pulverizadas y posteriormente analizadas para determinar la concentración de elementos mayores y algunos elementos traza por el método de Fluorescencia de Rayos X y la pérdida por calcinación. Adicionalmente también se analizaron dos muestras del volcán Ajusco y cinco muestras del volcán San Miguel. Estos análisis se llevaron a cabo en el Laboratorio Nacional de Geoquímica y Mineralogía, en el Instituto de Geología, Universidad Nacional Autónoma de México (UNAM), siguiendo la metodología descrita en Lozano-Santacruz y Bernal (2005). Los elementos traza y de las tierras raras fueron determinados en cinco muestras de la PBT y en las muestras del volcán Ajusco y el volcán San Miguel por el método de ICP-MS, en el Centro de Geociencias, UNAM, Campus Juriquilla, Querétaro, siguiendo la metodología descrita en Mori et al. (2007). Los resultados químicos se presentan en la Tabla 2.

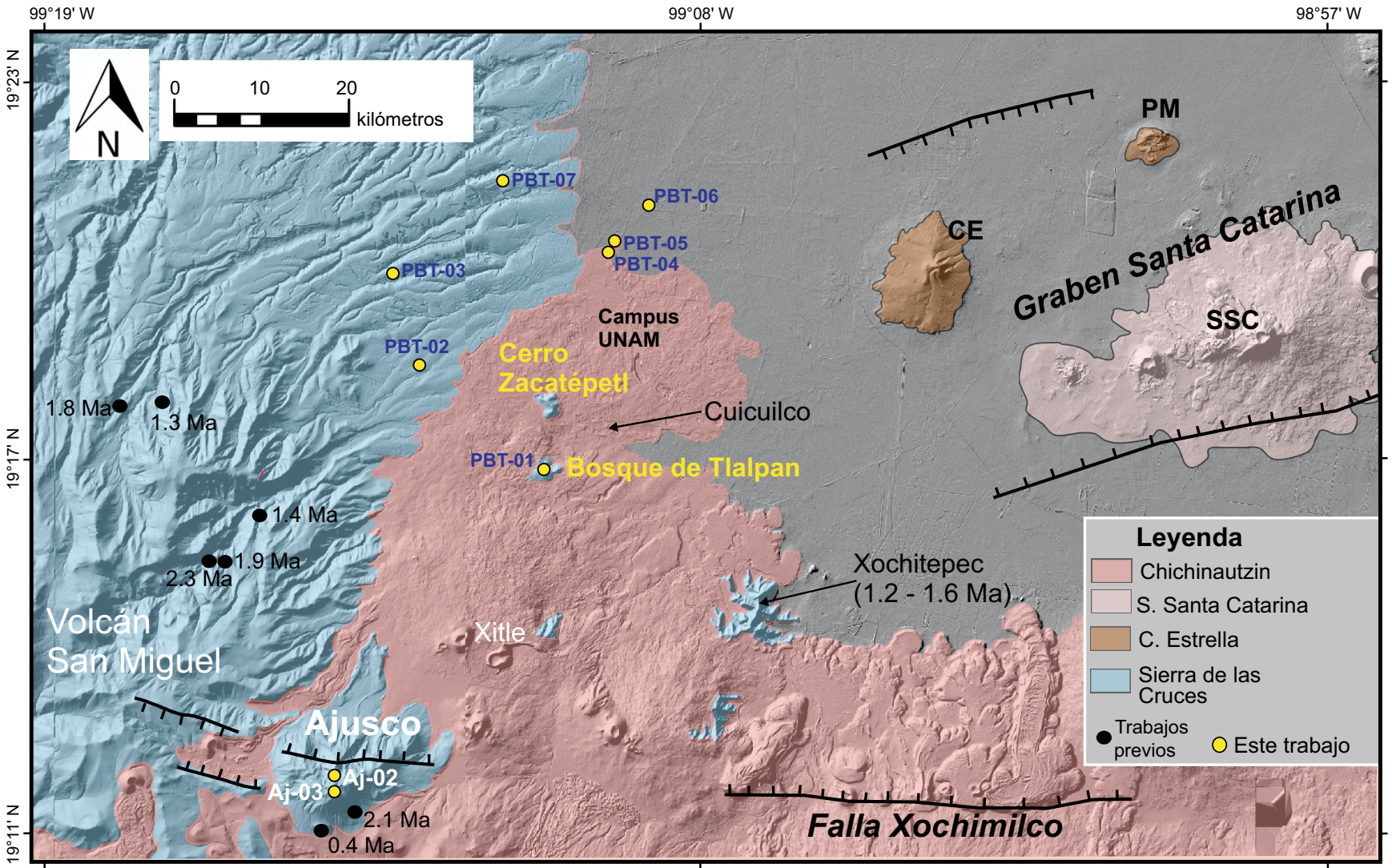

Figura 2. Mapa de la distribución de las distintas unidades litológicas de la parte sur de la cuenca de México (tomado de Arce et al., 2015) y la localización de los afloramientos que se describen en este trabajo. Las edades de los volcanes San Miguel y Ajusco fueron tomadas de Mejia et al. (2005), Mora-Alvarez et al. (1991), Osete et al. (2000), y Romero-Terán (2001). CE, Cerro de la Estrella; SSC, Sierra Santa Catarina; PM, Peñón del Marqués. La falla Xochimilco y fallas en los volcanes San Miguel y Ajusco fueron propuestas por García-Palomo et al. (2008) y la geometría de las fallas del graben de Santa Catarina fue retomada de Arce et al. (2013a). 
Tabla 1. Afloramientos de la pómez Bosque de Tlalpan descritos en este trabajo.

\begin{tabular}{|c|c|c|c|c|}
\hline \multirow[t]{2}{*}{ Muestra } & \multirow[t]{2}{*}{ Localidad } & \multicolumn{2}{|c|}{ Coordenadas } & \multirow{2}{*}{$\begin{array}{c}\text { Altitud } \\
\text { (m s.n.m.) }\end{array}$} \\
\hline & & Norte & Oeste & \\
\hline PBT-01 & Bosque de Tlalpan & $19^{\circ} 17^{\prime} 37.38$ & $99^{\circ} 11^{\prime} 53.02$ & 2,443 \\
\hline PBT-02 & Luis Cabrera & $19^{\circ} 19^{\prime} 13.06$ & $99^{\circ} 13^{\prime} 51.71$ & 2,369 \\
\hline PBT-03 & Av. de las Torres & $19^{\circ} 20^{\prime} 30.87$ & $99^{\circ} 14^{\prime} 23.31$ & 2,481 \\
\hline PBT-04 & Av. Universidad & $19^{\circ} 20^{\prime} 29.84$ & $99^{\circ} 10^{\prime} 55.13$ & 2,274 \\
\hline PBT-05 & C.C. Oasis & $19^{\circ} 20^{\prime} 43.84$ & $99^{\circ} 10^{\prime} 45.17$ & 2,268 \\
\hline PBT-06 & C.C. Coyoacán & $19^{\circ} 21^{\prime} 33.30$ & $99^{\circ} 10^{\prime} 17.40$ & 2,260 \\
\hline PBT-07 & Lomas de Tarango & $19^{\circ} 21^{\prime} 43.10^{\prime \prime}$ & $99^{\circ} 13^{\prime} 2.93^{\prime \prime}$ & 2,263 \\
\hline AJU-1602 & Volcán Ajusco & $19^{\circ} 12^{\prime} 39^{\prime \prime}$ & $99^{\circ} 15^{\prime} 42^{\prime \prime}$ & 3,822 \\
\hline AJU1603 & Volcán Ajusco & $19^{\circ} 12^{\prime} 28^{\prime \prime}$ & $99^{\circ} 15^{\prime} 31^{\prime \prime}$ & 3,908 \\
\hline SM1319 & Volcán San Miguel & $19^{\circ} 15^{\prime} 54^{\prime \prime}$ & $99^{\circ} 18^{\prime} 18^{\prime \prime}$ & 3,499 \\
\hline SM1339 & Volcán San Miguel & $19^{\circ} 17^{\prime} 59^{\prime \prime}$ & $99^{\circ} 18^{\prime} 18^{\prime \prime}$ & 3,436 \\
\hline SM-1325 & Volcán San Miguel & $19^{\circ} 13^{\prime} 45^{\prime \prime}$ & $99^{\circ} 18^{\prime} 43^{\prime \prime}$ & 3,494 \\
\hline SM-1327 & Volcán San Miguel & $19^{\circ} 13^{\prime} 18^{\prime \prime}$ & $99^{\circ} 18^{\prime} 58^{\prime \prime}$ & 3,617 \\
\hline SM-1313 & Volcán San Miguel & $19^{\circ} 14^{\prime} 13^{\prime \prime}$ & $99^{\circ} 23^{\prime} 19^{\prime \prime}$ & 3,054 \\
\hline
\end{tabular}

Claves de muestras: PBT, pómez Bosque de Tlalpan; AJU, volcán Ajusco; SM, volcán San Miguel. Abreviaturas: C.C., centro comercial; Av., avenida.

\section{Radiocarbono}

La medición por AMS de las tres muestras de paleosuelo, se realizó en grafito producido por la reducción de una muestra de $\mathrm{CO}_{2}$ sobre una catálisis de cobalto. El $\mathrm{CO}_{2}$ se obtuvo de la combustión de la muestra a más de $800^{\circ} \mathrm{C}$ en una atmósfera con un $100 \%$ de oxígeno. $\mathrm{El} \mathrm{CO}_{2}$ se secó con metanol/hielo seco y se colectó en nitrógeno líquido para su subsecuente reacción de grafitización.

El resultado analítico ("BP" o "pMC") se obtiene al medir la razón C14/C13 de la muestra relativo al C14/C13 en ácido oxálico II (NIST4990C) en uno de los aceleradores de partículas y utilizando una fuente de iones SNICS. Las muestras de aseguramiento de calidad (QA) se miden junto a las muestras incógnitas y cuentan con su propio informe de aseguramiento de calidad. El resultado de la datación por AMS es corregido en función del fraccionamiento total utilizando el valor d13C del grafito mecanizado. Para mayores detalles de la metodología visitar la página de Beta Analytics (https://www.radiocarbon.com/espanol/ ema-datacion-laboratorio.htm)

\section{Termoluminiscencia (TL)}

Algunos sólidos cristalinos al ser sometidos con radiación ionizante y posteriormente calentados por debajo de su temperatura de incandescencia, emiten fotones; a este fenómeno se le conoce como termoluminiscencia (TL). Este fenómeno de la termoluminiscencia tiene aplicaciones para la datación de muestras geológicas y arqueológicas (Aitken, 1985), utilizando minerales como el cuarzo, olivino, plagioclasa y adicionalmente vidrio. El principio de fechamiento radica en determinar el último evento de calentamiento al que fue sometida la muestra, a partir de que la muestra se enfría; ésta interactúa con la radiación natural proveniente de los radioisótopos más abundantes en la naturaleza $\left({ }^{238} \mathrm{U},{ }^{232} \mathrm{Th} y{ }^{40} \mathrm{~K}\right)$ más la dosis cósmica, acumulándose una señal luminiscente debido a los cambios energéticos que sufren los electrones de valencia en la estructura del sólido. En el laboratorio la muestra es calentada y se mide la señal luminiscente natural (TLN) emitida. La ecuación para determinar la edad por termoluminiscencia está dada por:

$$
\text { Edad } T L=\frac{\text { Paleodosis }}{\text { Tasa de dosis anual }}
$$

Edad $T L=\frac{\text { Dosis equivalente } Q+\text { factor por supralinealidad } l}{\text { Dosis alfa }+ \text { Dosis beta }+ \text { Dosis gama }+ \text { Dosis cósmica }}$
Donde la paleodosis [Gy] es la dosis acumulada por la muestra a través del paso del tiempo; a su vez la paleodosis se descompone en la dosis equivalente $\mathrm{Q}$ y el factor por supralinealidad I. La tasa de dosis anual [Gy/año] es la dosis de origen natural que la muestra recibe en el intervalo de un año y que está compuesta por las dosis alfa, beta, gama y cósmica. Existen otros factores que integran la ecuación como el factor de eficiencia alfa/beta y el factor de atenuación higroscópica.

En el punto de muestreo (sitio PBT-01) se colocó un espectrómetro gama portátil "Geofyzika Brno Portable Gamma Ray Spectrometer GS512 " con detector de NaI para la medición in situ de la concentración de los radioisótopos ${ }^{238} \mathrm{U}$, ${ }^{242} \mathrm{Th} y{ }^{40} \mathrm{~K}$ en el depósito de pómez, para calcular la tasa de dosis anual. Del punto de muestreo se colectaron aproximadamente dos kilogramos de muestra. El vidrio amorfo es el principal componente de la pómez, el cual es uno de los sólidos cristalinos con un espectro termoluminiscente óptimo para ser datado, razón por la cual se decidió concentrar el vidrio y eliminar el resto de impurezas o material lítico. Para esto la muestra de pómez fue sometida a flotación para separar y eliminar parte del material lítico por densidad, posteriormente la pómez fue triturada y tamizada en un intervalo de 100 y 300 micras, para ser tratada posteriormente con un separador magnético Frantz LB- ${ }^{\circledR}$, hasta alcanzar una concentración por arriba del $95 \%$ de pureza del vidrio. Posteriormente la muestra fue pulverizada en un mortero de ágata y separada en un tamaño de grano entre 4 y 11 micras (técnica de grano fino). Con esta muestra se prepararon 80 alícuotas de aproximadamente $2 \mathrm{mg}$. Las muestras fueron analizadas en un equipo lector de termoluminiscencia Daybreak 1100 Automated TL System seleccionando la combinación de filtros Corning 7-59 y Schott BG 12 para realizar las lecturas. Para calcular la paleodosis se empleó el método aditivo, que consistió en irradiar las muestras a diferentes dosis de radiación beta con una fuente de ${ }^{90} \mathrm{Sr}$, en un equipo Daybreak 801 Multiple Sample Irradiator. La temperatura de evaluación de la paleodosis fue entre 290 y $300^{\circ} \mathrm{C}$. Todos los análisis se elaboraron en el Laboratorio de Termolumiscencia, del Instituto de Geofísica, UNAM.

\section{GEOLOGÍA LOCAL}

La geología superficial del sur de la cuenca de México está representada por tres sierras volcánicas principales (Figura $1 \mathrm{~b}$ ), sin 


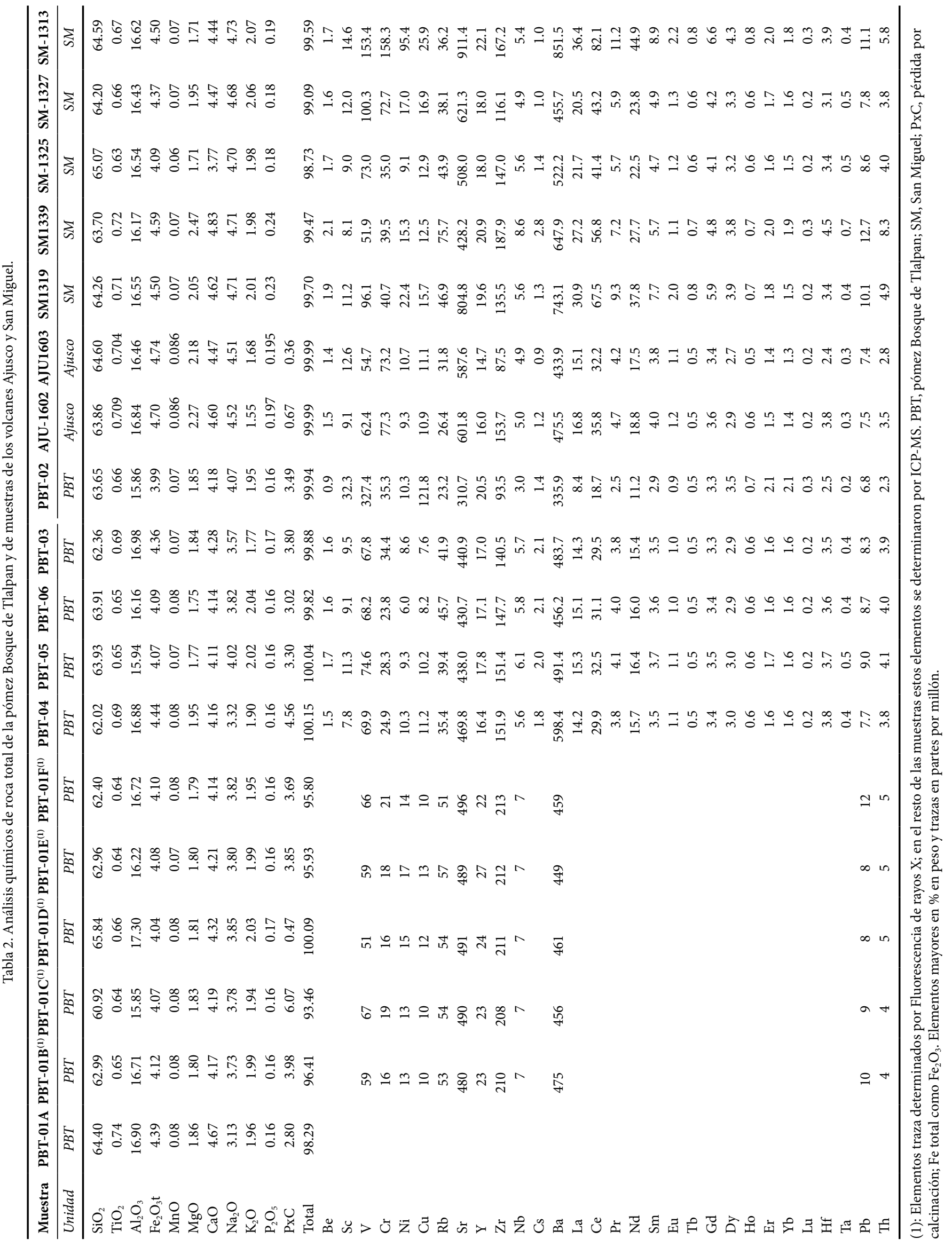


embargo, de acuerdo con perforaciones profundas se sabe que las rocas más antiguas por debajo del relleno volcánico, son rocas marinas de la Formación Morelos, de edad Cretácica (Fries, 1956, 1960; Mooser, 1963, 1975; De Cserna et al., 1988), descritas en los pozos profundos Mixhuca 1 y Tulyehualco 1 (Figura 1b) (PEMEX, 1987; Pérez-Cruz, 1988). Afloramientos superficiales de estas rocas marinas se distribuyen ampliamente en el Estado de Morelos.

Para la parte volcánica más antigua, dentro de la cuenca se han descrito rocas del Mioceno en el pozo San Lorenzo Tezonco, con edades desde 20.1 Ma (950 m de profundidad) hasta $13.5 \mathrm{Ma}(2,000 \mathrm{~m}$ de profundidad) (Arce et al., 2015), que se han correlacionado con la Formación Tepoztlán (Lenhardt et al., 2010; Arce et al., 2015), así como con la Sierra de Guadalupe de 13-14 Ma de edad (Lozano-Barraza, 1968), ubicada al norte de la Ciudad de México (Figura 1b).

La Sierra de Las Cruces está conformada por ocho estructuras volcánicas traslapadas (García-Palomo et al., 2008), cuyas edades varían de 3.7 Ma para la parte norte (Caldera La Catedral; Aguirre-Díaz et al., 2006), hasta 0.9 Ma para la parte sur (volcán Zempoala; Arce et al., 2008) (Figura 1b). Dentro de esta sierra se encuentran los volcanes poligenéticos San Miguel y Ajusco (Figuras 1b, 2), cuyas edades reportadas oscilan entre 2.3 y $0.4 \mathrm{Ma}$ (Mora-Alvarez et al., 1991; Osete et al., 2000; Mejia et al., 2005). Estos dos volcanes están conformados por derrames de lava, domos de lava, depósitos piroclásticos y lahares de composición andesítica y dacítica (Romero-Terán, 2001; Arce et al., 2008, 2015).

En la parte noreste del volcán San Miguel se observan abanicos constituidos por secuencias de depósitos piroclásticos diversos y depósitos de flujos de escombros intercalados (Arce et al., 2015) de edad desconocida, con un drenaje bien desarrollado y asociado al vulcanismo de la Sierra de Las Cruces (Figura 2). Los productos de la Sierra de Las Cruces se extendieron hacia el centro de la cuenca, como se identificó en la secuencia del pozo profundo San Lorenzo Tezonco entre las profundidades de 875 y $580 \mathrm{~m}$ y con edades de $5 \mathrm{a} 0.9 \mathrm{Ma}$ (Arce et al., 2013a, 2015). Además, se han descrito relieves aislados como el cerro Zacatépetl, Parque Bosque de Tlalpan, cerro Xochitepec, entre otros (Figura 2) que son contemporáneos con los volcanes Ajusco y San Miguel y están rodeados por productos lávicos del vulcanismo monogenético de la Sierra Chichinautzin. Estos cuerpos aislados fueron fechados entre 1.2 y 1.6 Ma (Arce et al., 2015), que se encuentran en el mismo rango de edades de los volcanes Ajusco y San Miguel.

La Sierra Nevada se localiza en la parte este de la cuenca de México y está conformada del norte hacia el sur por los estratovolcanes Tláloc, Telapón, Iztaccíhuatl y Popocatépetl (Figura 1b). Las edades para esta sierra son variables; para el Tláloc y Telapón se han reportado edades desde 1.2 Ma (Cadoux et al., 2011; Macias et al., 2012), para el Iztaccíhuatl se han fechado rocas en 1.1 a $0.45 \mathrm{Ma}$ (Nixon, 1989; Macias et al., 2012) mientras que para el Popocatépetl las rocas más antiguas han sido fechadas en 0.33 Ma (Sosa-Ceballos et al., 2015), representadas por la estructura denominada El Ventorrillo, la cual fue anterior al Popocatépetl moderno. En los pozos profundos no se han logrado identificar rocas de esta sierra volcánica, sin embargo se ha reportado la Pómez Tutti Frutti en el campo volcánico Chichinautzin, así como en algunos pozos someros y obras civiles de la Ciudad de México (Siebe y Macías, 2004; Lozano-García, 1989; Lozano-García y Ortega-Guerrero, 1998).

Finalmente, el borde sur de la cuenca de México está conformada por el campo volcánico Chichinautzin, el cual consta de varias estructuras monogenéticas, con edades desde 1.2 Ma (Arce et al., 2013b) hasta 1,600 años antes del presente (A.P.) (Siebe, 2000). Resalta la presencia de derrames de lava muy viscosos, aparentemente fisurales, asociados a la Falla Xochimilco (Figura 2) (García-Palomo et al., 2008). Tanto al Cerro de la Estrella, al Peñón del Marqués y Sierra de Santa Catarina
(Figura 2), se les puede considerar como parte del vulcanismo del campo volcánico Chichinautzin, con base en la composición química, tipo de vulcanismo e intervalo de edad (Arce et al., 2013b; 2015).

Los depósitos lacustres han sido estudiados a través de núcleos de pozos someros en el lago de Chalco, de aproximadamente $30 \mathrm{~m}$, en donde se observa una intercalación de sedimentos lacustres y depósitos volcánicos como registro de la estratigrafía del Holoceno y Pleistoceno (Lozano-García y Ortega-Guerrero, 1998; Caballero y Ortega-Guerrero, 1998), así como de las condiciones paleoambientales. Las edades reportadas para estos pozos someros oscilan entre 10 y 220 mil años (Lozano-García y Ortega-Guerrero, 1998; Lozano-García et al., 1993; Brown et al., 2012).

\section{AFLORAMIENTOS DE LA PÓMEZ BOSQUE DE TLALPAN}

En total se describieron siete afloramientos (Tabla 1; Figura 2), cuatro de éstos en superficie, uno localizado en el Bosque de Tlalpan (PBT-01), al sur de la Ciudad de México (Figuras 2 y 3a), Delegación Tlalpan, otro más en la zona de Luis Cabrera (PBT-02; Figura 3b), el tercero en la supervía Poniente (PBT-03), en la Delegación Magdalena Contreras (Figuras 2 y $3 c$ ) y el cuarto en Lomas de Tarango (PBT-07), delegación Magdalena Contreras. Los otros afloramientos fueron descritos en excavaciones de obras civiles (Figura 4), uno de éstos localizados en Av. Universidad, en la Colonia Oxtopulco (PBT-04) $\mathrm{y}$ otro en donde actualmente se encuentra el centro comercial Oasis (PBT-05) (Av. Universidad y Av. Miguel Ángel de Quevedo), ambos en la Delegación Coyoacán (Tabla 1); el tercer afloramiento aún sigue en obra y se ubica al este del centro comercial Plaza Coyoacán, en la Delegación Benito Juárez (PBT-06; Figura 4c).

De manera general, la pómez Bosque de Tlalpan es un depósito de pómez de color blanco, constituido por aproximadamente $90 \%$ en volumen de fragmentos de pómez subangulosos y vesiculares, soportados grano-grano, del tamaño del lapilli grueso hasta algunos bloques (Figura 5). También se observan algunos fragmentos densos de lava alterada (componentes accidentales) que tienen un tamaño promedio de $3.5 \mathrm{~cm}$ de diámetro (promedio de cinco fragmentos) en el afloramiento PBT-01. Así mismo se pueden observar algunos cristales de biotita en la matriz del depósito. En general el depósito es masivo, aunque en ocasiones se logra apreciar una concentración de clastos más grandes a la mitad del depósito, con líticos densos de hasta $13 \mathrm{~cm}$ de diámetro, los cuales probablemente son fragmentos balísticos que pueden sugerir una cercanía relativa con la fuente de emisión.

El espesor máximo fue medido en el afloramiento del Bosque de Tlalpan (PBT-01) en donde alcanza los $3.0 \mathrm{~m}$, sin embargo, no fue posible observar la parte superior del depósito ya que éste se encuentra sobreyacido por material de retrabajo, lo que sugiere que fue parcialmente erosionado. No obstante, en otros afloramientos como en PBT-02 y PBT-03 (Figuras 3a, 3b y 6), se observó que este depósito de caída de pómez, cambia hacia su cima de manera gradual a una ceniza gruesa, media y hasta fina, sobreyacida por varias capas de $20 \mathrm{~cm}$, de espesor constante y con buen grado de selección, lo que sugiere que se trata de depósitos de caída de ceniza y lapilli fino (Figuras $4 b, 5$ c y 6). Estas capas de ceniza y lapilli fino fueron observados en los afloramientos PBT-02, PBT-04 y PBT-06 (Figura 6) y en total suman un espesor de hasta $60 \mathrm{~cm}$ (medido en el afloramiento PBT-02).

En el afloramiento del Bosque de Tlalpan (PBT-01), la PBT sobreyace a un depósito de color café claro, masivo, con algunos poros, constitutido por escasos fragmentos de pómez y líticos del tamaño del lapilli, dentro de una matriz de ceniza fina, parcialmente convertido a suelo (Figura 5). Este horizonte fue catalogado como material de retrabajo, y de él se obtuvo una muestra total para fechamiento de la materia 


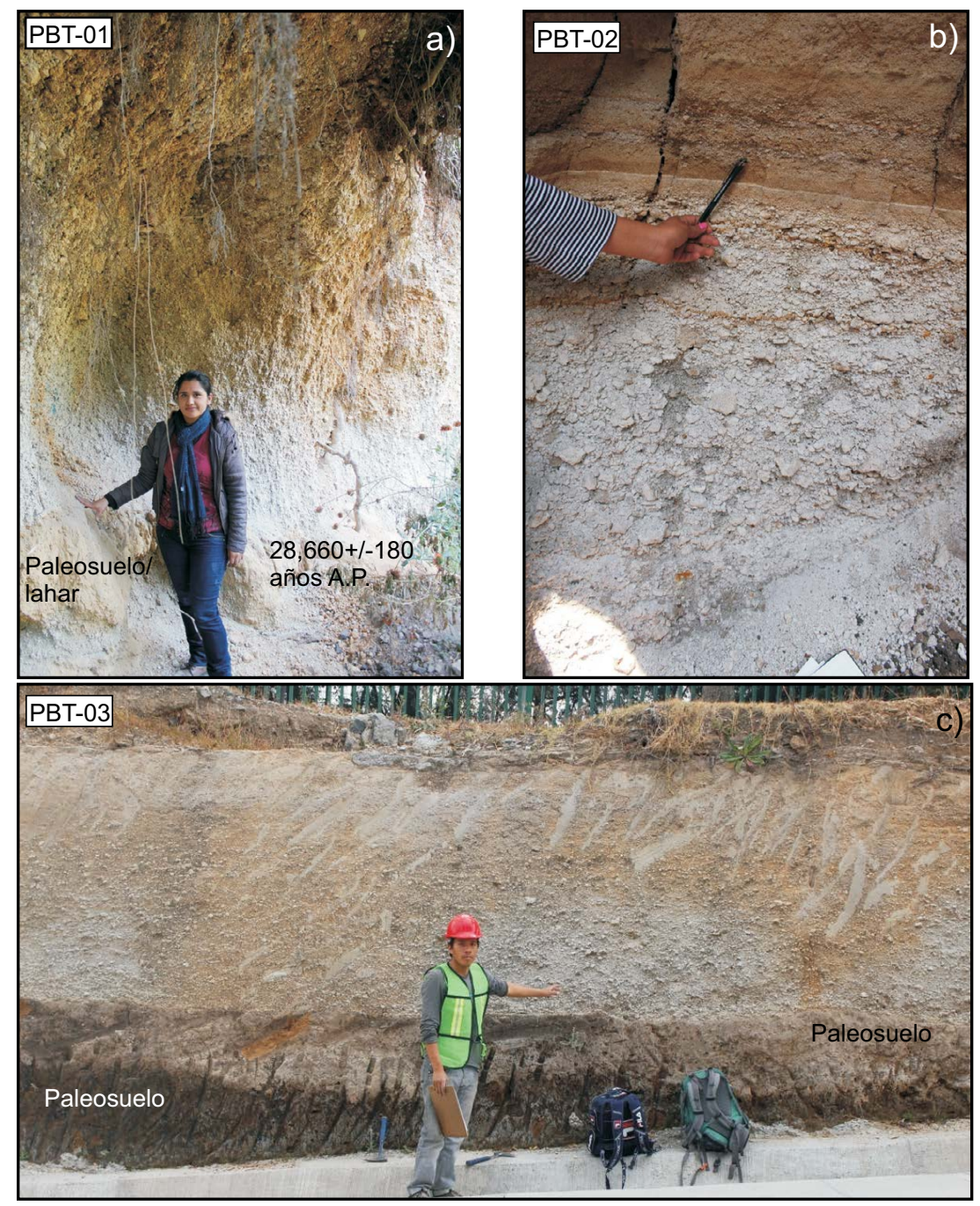

Figura 3. a) Fotografía del afloramiento PBT-01 en donde se aprecia el depósito de pómez de caída, un paleosuelo fechado en este trabajo y un segundo depósito de caída de pómez; b) Afloramiento PBT-02, en donde se aprecia la parte superior del depósito de la pómez Bosque de Tlalpan, el cual presenta una terminación con ceniza fina, sobreyacido por capas de ceniza gruesa a fina del mismo depósito; c) Fotografía del afloramiento PBT-03, en donde se aprecia el depósito de caída, subyacido por un paleosuelo de color oscuro y sobreyacido por material de retrabajo.

orgánica por radiocarbono. Este material retrabajado yace sobre otro depósito de caída, de $50 \mathrm{~cm}$ de espesor (Figura 5), con dimensiones de pómez menores al depósito PBT, denominado de manera informal como Pómez con Anfíbol (Figura 6), el cual corresponde a otro evento eruptivo diferente a la PBT, ya que entre ambos se encuentra un paleosuelo que sugiere un tiempo considerable entre los dos eventos eruptivos. Debido a que ambos depósitos de caída están separados por el material retrabajado y parcialmente convertido a suelo, esto sugiere que no existe ninguna relación entre ambos depósitos.

Las características de color, espesor, dimensiones y abundancia de los fragmentos de pómez, aunado a la asociación mineralógica y composición química que se aborda más adelante, fueron clave para poder identificar a este depósito en otros sitios (Figura 6) y más aún, puede servir como marcador estratigráfico en toda la cuenca de México, incluso en perforaciones someras que se lleven a cabo en el futuro. La correlación de afloramientos (Figura 6) también sirvió para definir grosso modo el eje de dispersión de la pómez, la cual fue hacia el NE.

Por otro lado, se llevaron a cabo análisis sedimentológicos de las secciones PBT-01, PBT-04 y PBT-07 del depósito de caída PBT (Figura 5).
El histograma de frecuencia de la muestra basal es unimodal, con un diámetro mediano de grano $(\mathrm{Md} \varphi)$ de -2.2 y un grado de selección $(\sigma \varphi)$ de 1.9; mientras que para la parte media, el histograma despliega una forma bimodal, con un $\operatorname{Md} \varphi=-3.2$ y un $\sigma \varphi=2.4$. Los constituyentes de la PBT en general son fragmentos de pómez, con muy escasos fragmentos accidentales (lavas alteradas probablemente dacíticas, de color rojo y gris oscuro) con alteración hidrotermal, y vidrio para las fracciones más finas (Figura 5).

\section{PETROGRAFÍA Y GEOQUÍMICA}

Se realizaron en total cinco láminas delgadas de fragmentos de pómez del depósito PBT y se llevaron a cabo 12 análisis químicos de roca total (Tabla 2). Debido a que la composición química y mineralógica de la pómez es homogénea se realizó el conteo modal solo de dos láminas delgadas. De manera general la pómez es hipocristalina, porfídica (Figura 7), con una cristalinidad relativamente baja, que oscila entre 9 y $12 \%$ vol., con una mineralogía representada en orden de abundan- 

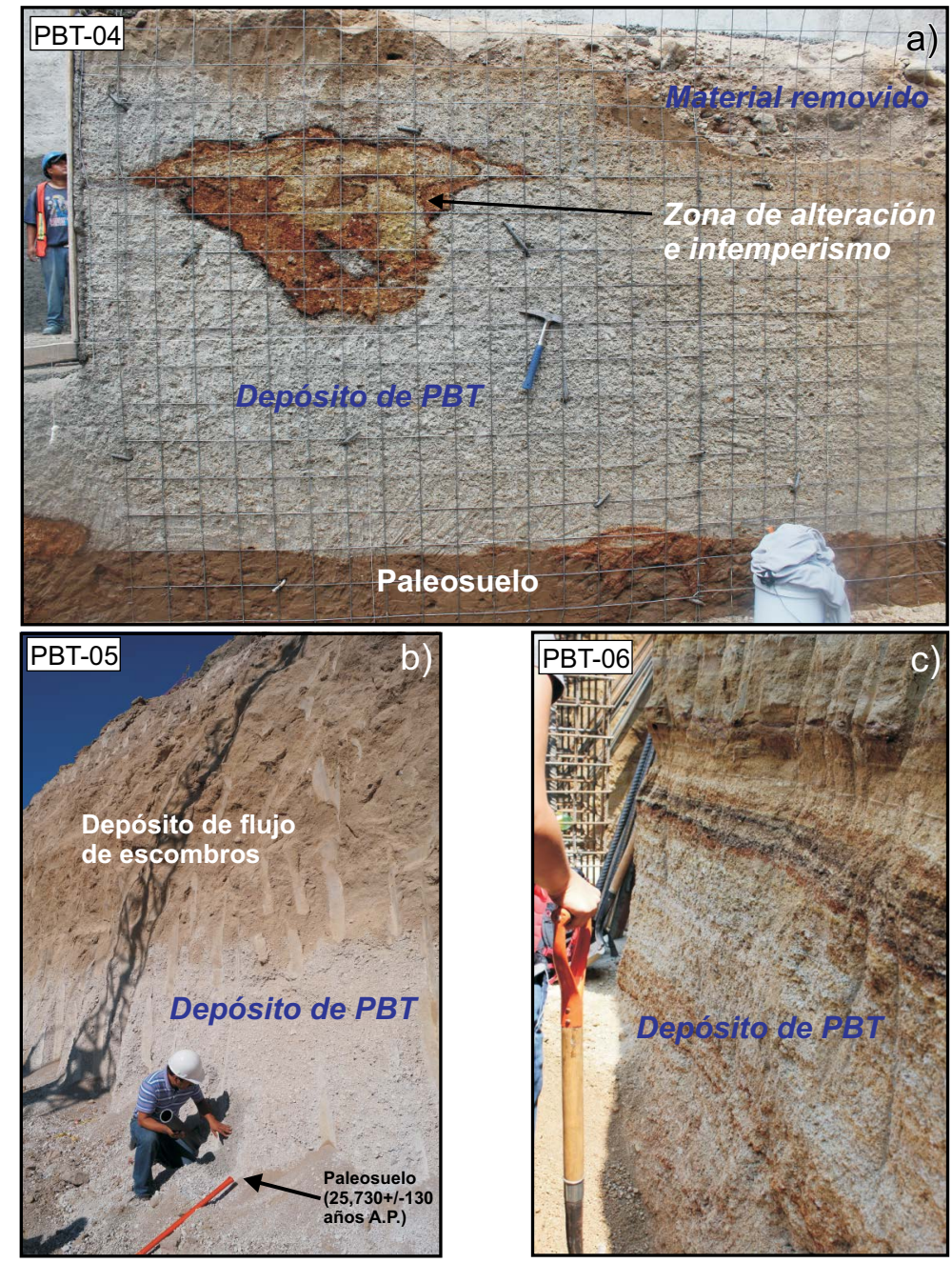

Figura 4. a) Fotografía del afloramiento PBT-04 en donde se aprecia el depósito de pómez que sobreyace a un paleosuelo café oscuro y subyace a material de retrabajo. Nótese la zona de alteración por intemperismo; b) Fotografía del afloramiento PBT-05, en donde se aprecia el depósito de la pómez Bosque de Tlalpan (PBT) sobreyacido por un depósito de flujo de escombros y subyacido por un paleosuelo el cual fue fechado por C-14; c) Fotografía del afloramiento PBT-06, en la cual se observa la parte superior del depósito PBT, que consta de varias capas de lapilli fino y ceniza gruesa.

cia por fenocristales $(>0.3 \mathrm{~mm})$ y microfenocristales $(0.03-0.3 \mathrm{~mm})$ de plagioclasa (4-6\% vol.), anfíbol (2-3\% vol.), ortopiroxeno ( $0.6 \%$ vol., biotita (1.3\% vol.), óxidos de Fe-Ti (1.4\% vol., presente sólo como microfenocristal), así como cuarzo de manera muy escasa ( $<1 \%$ vol.), además del circón como mineral accesorio, inmersos en una matriz vítrea (71-80 \% vol.) y vesicular (10-17\% vol.). En general los fenocristales presentan formas subédricas a anédricas, comúnmente con bordes corroídos y golfos de disolución (Figura 7a), así como textura esqueletal en las plagioclasas. El cuarzo comúnmente muestra corona de disolución, mientras que los microfenocristales presentan formas principalmente euédricas. También es común la presencia de fragmentos de cristales con formas irregulares y angulosas. Así mismo, se observan glomerocristales de ortopiroxeno y anfíbol (Figura 7e y 7f). El vidrio de la matriz combinado con las abundantes vesículas, conforman una estructura fluidal cuando las vesículas son alargadas, pero en otros casos éstas son esféricas.

Los análisis químicos de roca total se llevaron a cabo utilizando fragmentos de pómez de todos los afloramientos de la PBT, así como cinco muestras de lavas dacíticas del volcán San Miguel y dos de lavas dacíticas del volcán Ajusco para fines comparativos (Tabla 2). De acuerdo con los resultados obtenidos, la pómez tiene una composición homogénea (Figura 8 y Tabla 2), con 64.9 a $66 \%$ en peso de $\mathrm{SiO}_{2}$ (en base anhidra) que cae dentro del campo de las dacitas, de afinidad calcialcalina (Figura $8 \mathrm{a}$ ), con contenido moderado de potasio $(\sim 2 \%$ en peso). En los diagramas binarios se aprecian correlaciones débiles negativas en $\mathrm{CaO}$ y $\mathrm{Fe}_{2} \mathrm{O}_{3}$ y positivas en $\mathrm{Na}_{2} \mathrm{O}$. Por cuestiones de comparación, en la Figura 8 también se grafican datos de dacitas de los volcanes Ajusco y San Miguel, debido a que son candidatos como fuente de emisión de la erupción que dio origen a la PBT. Sin embargo, los resultados demuestran que no existe un traslape claro de las dacitas de ambos volcanes con las muestras de la PBT. Esto no es raro puesto que los volcanes poligenéticos comúnmente presentan variaciones composicionales grandes debido a los procesos de evolución magmática que tiene lugar en los reservorios magmáticos, por lo tanto no es posible aseverar ni descartar la relación genética entre el depósito PBT y alguno de estos dos volcanes.

De acuerdo con las gráficas multielementales, se aprecia un enriquecimiento relativo en elementos incompatibles y empobrecimiento en elementos compatibles (Figura 9a), con marcadas anomalías negativas en Nb, Ta y Ti. Así mismo se observa una disminución progresiva 

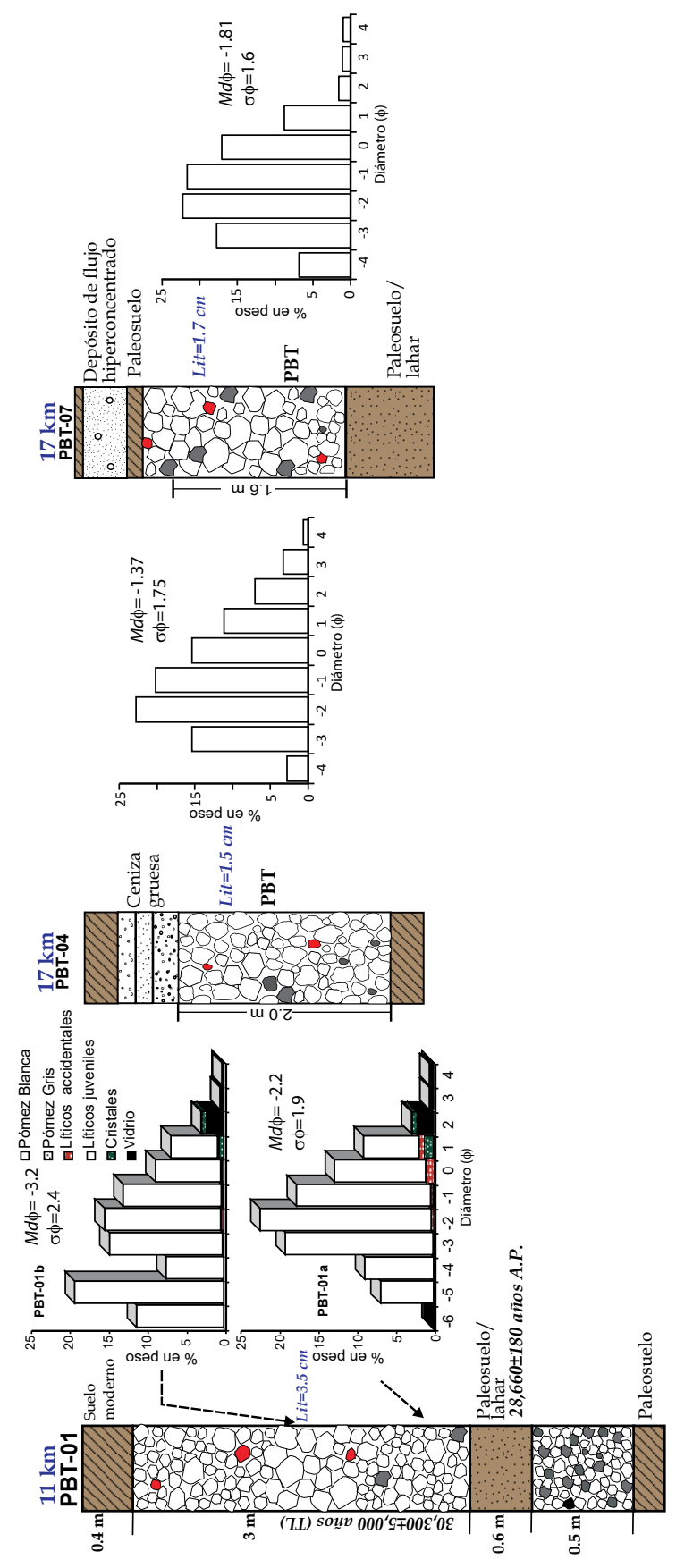
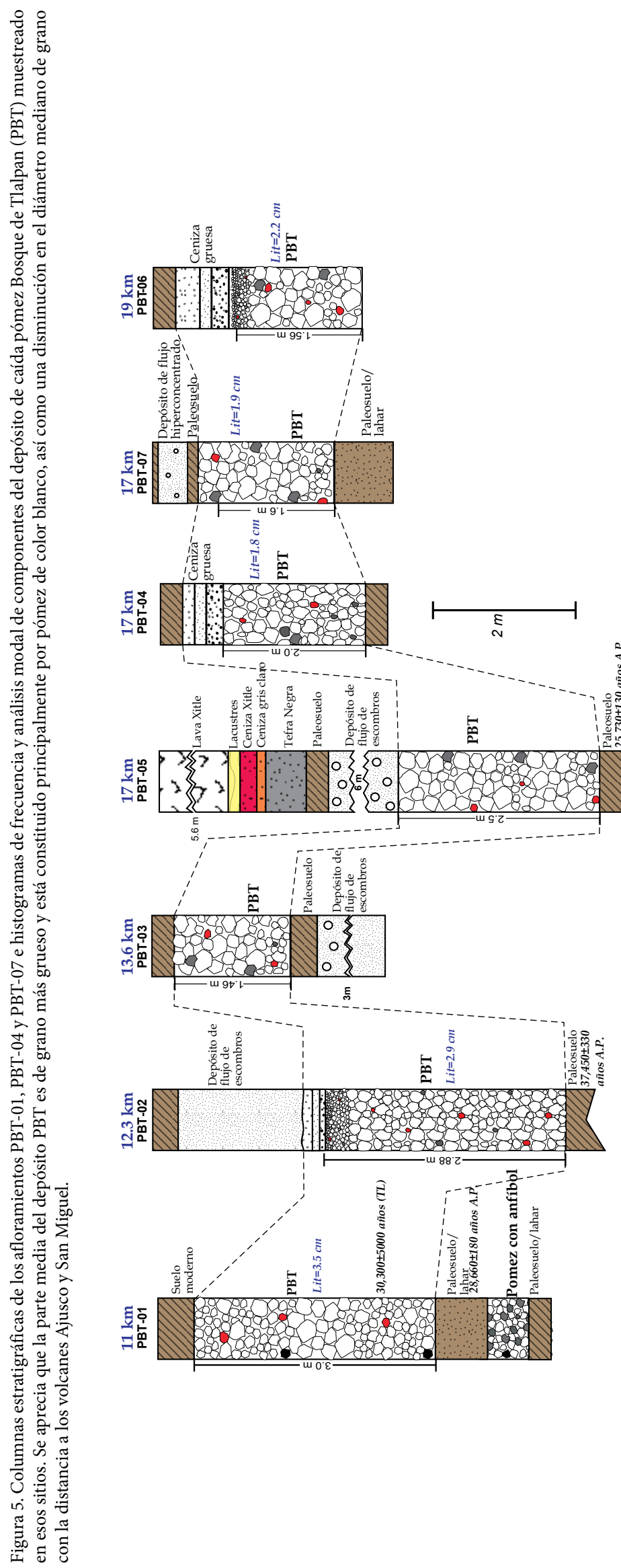

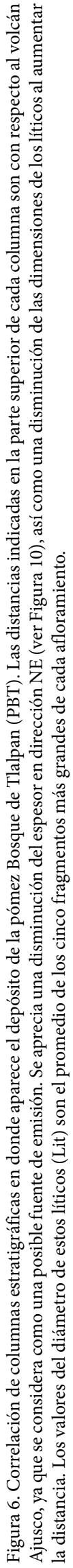


de tierras raras ligeras hacia las tierras raras pesadas, con una ligera anomalía negativa en Eu (Figura 9b). Estos patrones son típicos de ambientes de subducción, principalmente las anomalías negativas en $\mathrm{Nb}$ y $\mathrm{Ta}$, ya que estos elementos no son movilizados por fluidos acuosos (Saunders et al., 1980).

\section{FECHAMIENTO DE LA PBT}

En total se obtuvieron tres fechamientos por el método de radiocarbono (por el método AMS) de paleosuelos colectados directamente debajo del depósito PBT (Figura 6). El paleosuelo del afloramiento PBT-01 es de color café claro, masivo, con algunos fragmentos de pómez y arrojó una edad de 28,660 \pm 180 años A.P. (edad convencional). El segundo paleosuelo fechado se muestreó en el afloramiento PBT-02 (Tablas 1 y 3), el cual es de color café claro, masivo con un espesor mínimo de $20 \mathrm{~cm}$, que arrojó una edad de 37,450 \pm 330 años A.P. (edad convencional). El tercer paleosuelo fue muestreado en el afloramiento PBT-05 (Figura 6; Tablas 1 y 3), de color café claro, masivo, con un espesor mínimo de $40 \mathrm{~cm}$ y arrojó una edad de 25,730 \pm 130 años A.P. (edad convencional).

Por otra parte el fechamiento que se llevó a cabo por termoluminscencia de la muestra PBT-01, arrojó los siguientes datos: la tasa de dosis anual se calculó a partir de las dosis alfa, beta y gama (Guérin et al., 2011) y la contribución de la dosis de radiación cósmica. Una vez calculada la paleodosis y la tasa de dosis anual, se sustituyeron los valores en la Ecuación 1. La paleodosis calculada fue de 57.66 \pm 9.64 Gy; la tasa de dosis anual fue calculada en $1.902 \times 10^{-3} \pm 0.095 \mathrm{~Gy} / \mathrm{año}$;
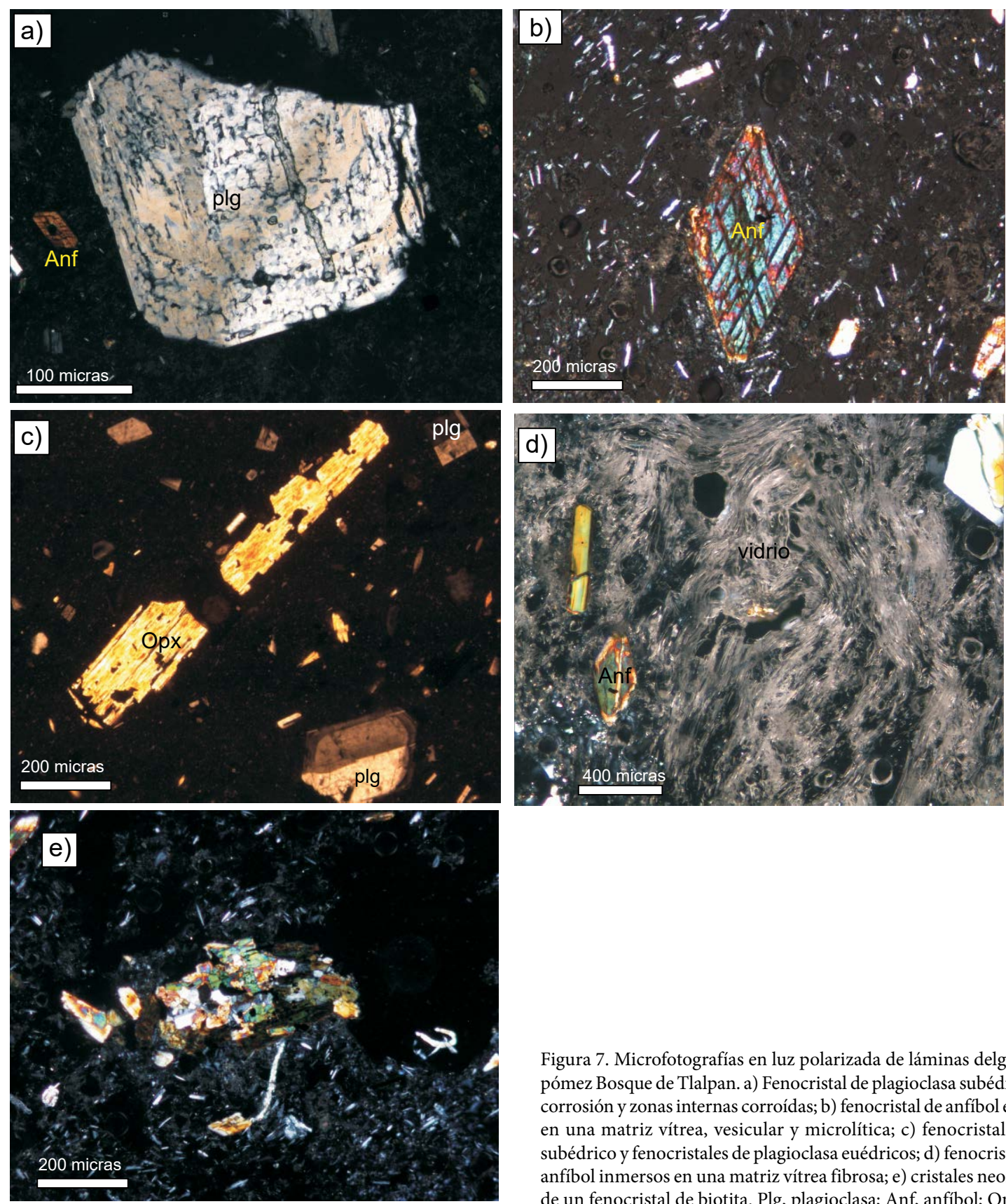

Figura 7. Microfotografías en luz polarizada de láminas delgadas del depósito pómez Bosque de Tlalpan. a) Fenocristal de plagioclasa subédrica, con golfos de corrosión y zonas internas corroídas; b) fenocristal de anfíbol euédrico, inmerso en una matriz vítrea, vesicular y microlítica; c) fenocristal de ortopiroxeno subédrico y fenocristales de plagioclasa euédricos; d) fenocristales euédricos de anfíbol inmersos en una matriz vítrea fibrosa; e) cristales neoformados a partir de un fenocristal de biotita. Plg, plagioclasa; Anf, anfíbol; Opx, ortopiroxeno. 

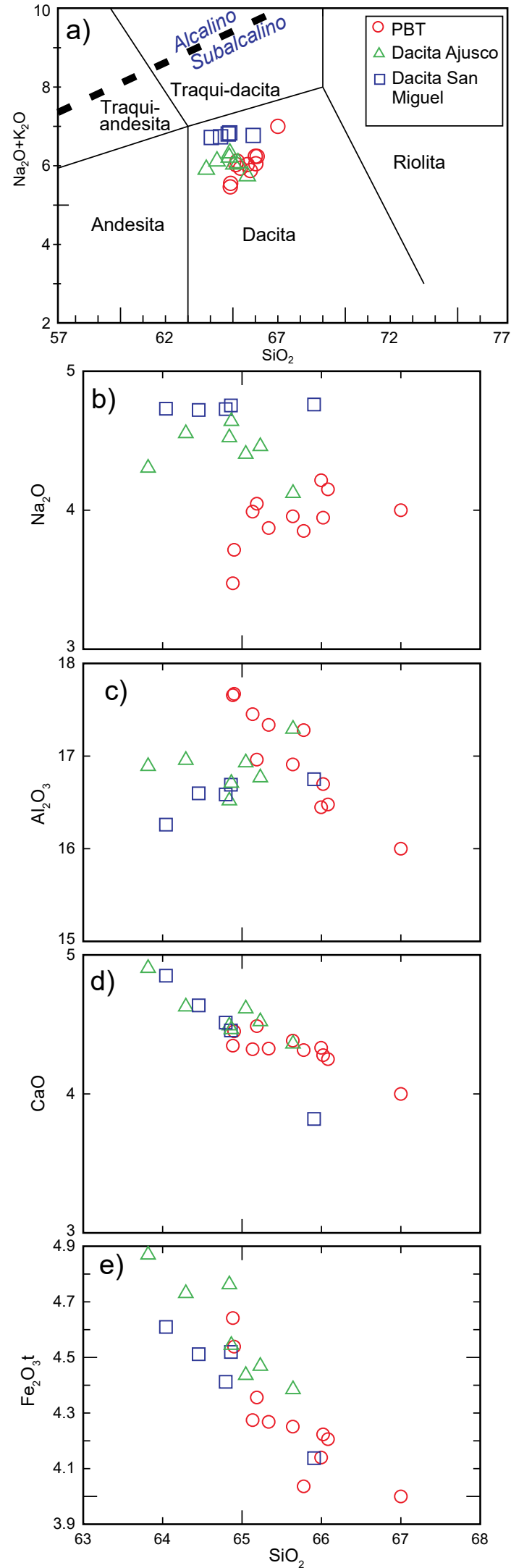

Figura 8. a) Diagrama de clasificación química (Le Bas et al., 1986) de álcalis totales $v$ s. sílice de las muestras de la pómez Bosque de Tlalpan (PBT) y de dacitas de los volcanes Ajusco y San Miguel; b-d) diagramas binarios de las mismas rocas analizadas, en donde se observan algunas tendencias composicionales.
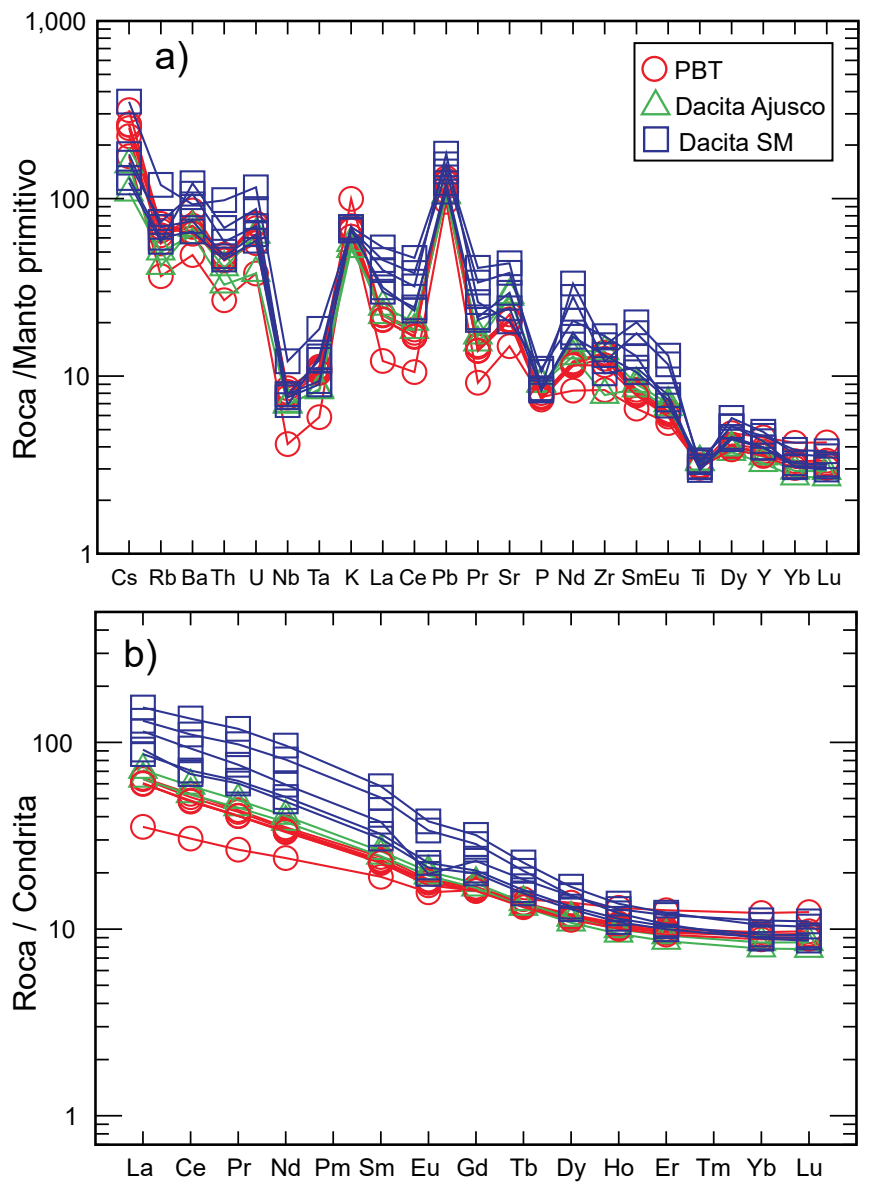

Figura 9. a) Diagrama multielemental normalizado a valores del manto primitivo (Sun y McDonough, 1989) de muestras de la pómez Bosque de Tlalpan (PBT) y de dacitas de los volcanes Ajusco y San Miguel, discutidos en el texto; b) diagrama de los elementos de las tierras raras normalizados con valores de condrita de Sun y McDonough (1989) del depósito PBT y dacitas de los volcanes Ajusco y San Miguel.

con lo cual se obtuvo una edad de 30,300 $\pm 5,000$ años. Esta edad está dentro del intervalo de edades obtenido por radiocarbono de los paleosuelos, por lo tanto representa una prueba más de que el depósito de caída Bosque de Tlalpan es del Pleistoceno Tardío.

\section{DISCUSIÓN}

\section{Edad de erupción de la pómez Bosque de Tlalpan}

Para determinar la edad de la pómez Bosque de Tlalpan se realizaron tres fechamientos por el método de radiocarbono y un fechamiento por el método de termoluminscencia. Para el radicocarbono se utilizaron muestras de paleosuelos, los cuales fueron colectados debajo del depósito PBT en los afloramientos PBT-01, PBT-02 y PBT-03 (Figuras 3 y 4); mientras que el de termoluminiscencia se realizó en vidrio de la muestra PBT-01.

Los análisis por radiocarbono arrojaron edades de 25,730 \pm 130 ; $28,660 \pm 180$ y 37,450 \pm 330 años A.P. (Tabla 3), aunado a la edad de $30,300 \pm 5,000$ años determinado por termoluminscencia, soportan la edad de entre 25 - 37 ka para este depósito. Además el depósito en superficie se observa muy bien preservado, a pesar de que la pómez es muy fácil de alterarse por intemperismo, lo cual sugiere también una edad relativamente joven. La variabilidad en edad de los paleosuelos es 


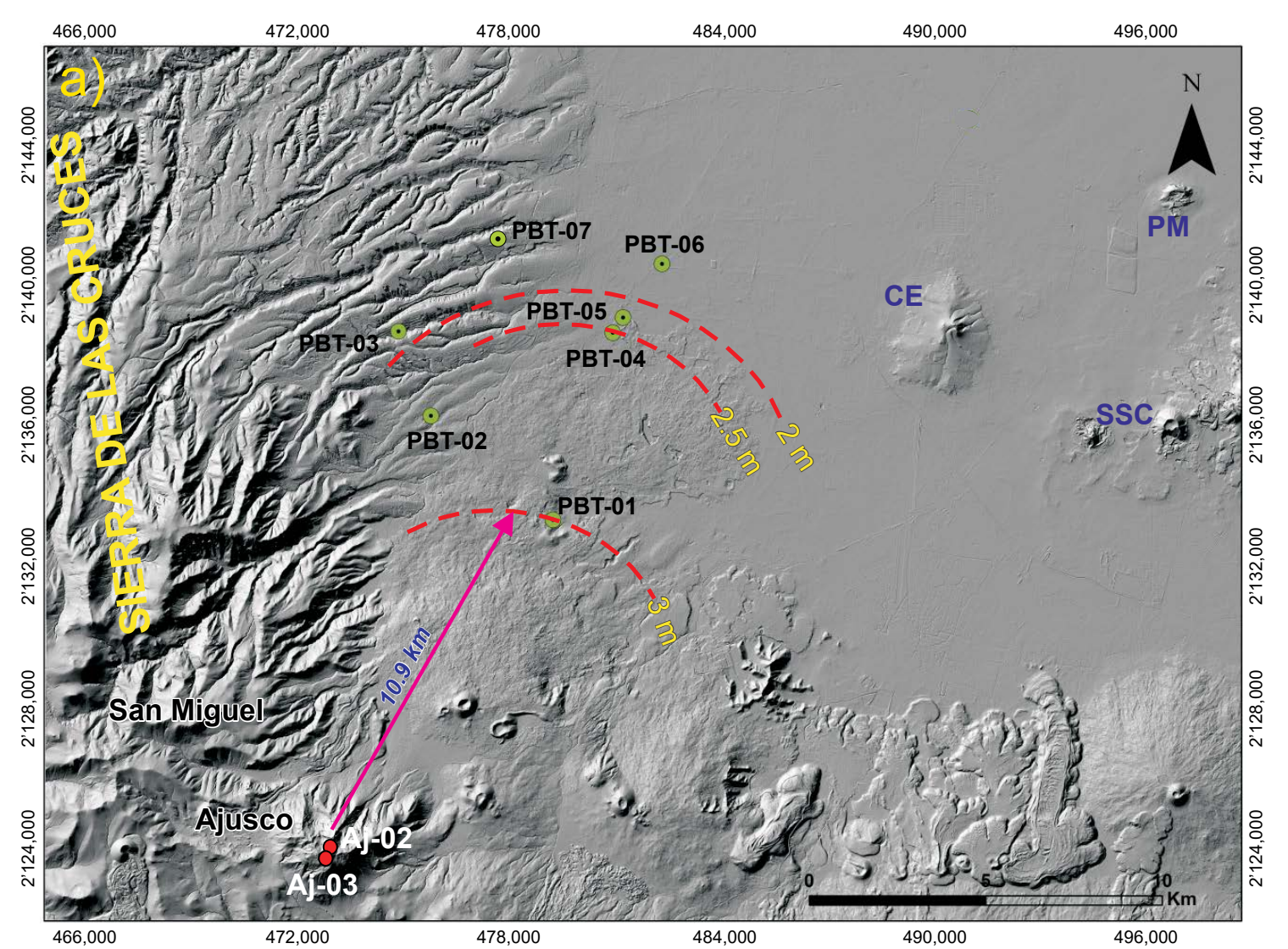

Figura 10. a) Modelo digital de elevación en donde se proponen isopacas preliminares del depósito de la pómez Bosque de Tlalpan, las cuales sugieren que la fuente de emisión estaría al SW de las mismas, en donde se ubica el volcán Ajusco y el volcán San Miguel. La isopaca de $3 \mathrm{~m}$ estaría ubicada a $10.9 \mathrm{~km}$ desde la cima del volcán Ajusco. CE, Cerro de la Estrella; SSC, Sierra Santa Catarina; PM, Peñón del Marqués. Aj-02 y Aj-03 representan la ubicación de las dos muestras analizadas del volcán Ajusco.

normal, dado que la pómez de caída pudo haber cubierto paleosuelos de distintas edades, sin embargo es posible plantear que la edad máxima más certera del depósito es de 25,730 \pm 130 años A.P.

\section{Fuente de emisión}

Debido a que se tienen pocos afloramientos, no fue posible construir mapas de isopacas e isopletas, las cuales permiten definir la fuente de dónde fueron expulsados los fragmentos de pómez (RodríguezElizarrarás et al., 2002). Sin embargo, dado que los espesores del depósito PBT son considerables ( $3 \mathrm{~m}$ ), así como los fragmentos de pómez de hasta $10 \mathrm{~cm}$ de diámetro y un promedio de líticos densos de 3.5 $\mathrm{cm}$ de diámetro en el afloramiento PBT-01 (Figura 6), sugieren que la fuente de emisión estuvo relativamente cerca. Cabe resaltar que en este mismo afloramiento se observaron algunos fragmentos líticos de 9 y 13 $\mathrm{cm}$ de diámetro, los cuales podrían representar fragmentos balísticos, ya que se salen del valor promedio y por lo tanto este afloramiento estaría próximo a la fuente de emisión. Además, de manera general se aprecia que los espesores y diámetros de los fragmentos de pómez y líticos disminuyen hacia el NE (Figuras 6 y 10), por lo tanto la fuente de emisión debería estar hacia el SW, en cuya dirección se encuentran los volcanes Ajusco y San Miguel (conforman parte de la Sierra de Las Cruces) y son los únicos volcanes poligenéticos en esta área. Debido a la composición dacítica y el tipo de material piroclástico (pómez), se descarta que la fuente de emisión sea un cono monogenético, ya que los volcanes monogenéticos generalmente producen fragmentos de escoria y son de composiciones más básicas (andesítica a andesítica basáltica). De acuerdo con los datos químicos de la PBT y los volcanes Ajusco y San Miguel, no es posible discernir cual de estos dos fue el centro de emisión de la pómez, ya que dichas composiciones no se traslapan ni en elementos mayores ni trazas. Sin embargo, las isopacas preliminares de la Figura 10 sugieren que la fuente de emisión de la PBT tuvo que ser un volcán poligenético, ya sea el volcán Ajusco

Table 3. Resultados del análisis por radiocarbono mediante Espectrómetro de Masas con Aceleradores de paleosuelos del depósito pómez Bosque de Tlalpan (PBT).

\begin{tabular}{lcccc}
\hline Sample & Material & $\begin{array}{c}\text { relación } \\
\text { C-13/C-12 }\end{array}$ & Edad convencional & Edad calibrada \\
\hline PBT-01 & Paleosuelo debajo de la PBT & 24.7 & $28,660 \pm 180$ años AP & 33,460 a 32,720 cal. a A.P. \\
PBT-02 & Paleosuelo debajo de la PBT & 18.1 & $37,450 \pm 330$ años AP & 42,373 a 41,379 cal. a A.P. \\
PBT-05 & Paleosuelo debajo de la PBT & 17.9 & $25,730 \pm 130$ años AP & 30,810 a 30,330 cal. a A.P. \\
\hline
\end{tabular}

Todas las muestras fueron colectadas debajo del depósito Pómez Bosque de Tlalpan. cal. a A.P., años calibrados antes del presente. 
o el volcán San Miguel y por lo tanto, la edad de este depósito entre $25,730 \pm 130$ a $37,450 \pm 330$ años A.P. representa la edad más joven reportada hasta ahora para la Sierra de Las Cruces. Así mismo, se descarta que la fuente de emisión sea el volcán Nevado de Toluca o Popocatépetl dado que el espesor y dimensiones de los clastos en todos los afloramientos, sugieren que la fuente está relativamente cerca. Como comparación, el depósito Pómez Toluca Superior del Nevado de Toluca en la cuenca de México tiene un espesor de aproximadamente $30 \mathrm{~cm}$ y es de tamaño de ceniza gruesa. Además este tipo de depósitos generalmente alcanza grandes distancias, por lo que es muy probable que se haya depositado en los antiguos lagos de Chalco y Texcoco, en donde se podría utilizar como un marcador estratigráfico.

\section{Estilo eruptivo}

Para poder determinar el estilo eruptivo de una erupción mediante los depósitos piroclásticos, se requiere de datos de isopacas e isopletas (Pyle, 1989), pero como ya se mencionó, debido a la restricción de afloramientos no fue posible hacer estos mapas para el depósito PBT. Sin embargo, el depósito presenta características similares a otros depósitos producidos por erupciones plinianas como la estructura masiva del depósito, constituido en su mayoría por fragmentos de pómez muy vesicular, así como los espesores y su distribución, a pesar de que son pocos afloramientos descritos, entre ellos hay una distancia de $8 \mathrm{~km}$ (Figura 10). Si consideramos que la fuente de emisión del depósito PBT es uno de los dos volcanes mencionados (Ajusco o San Miguel), el espesor máximo de $3 \mathrm{~m}$ estaría ubicado a una distancia de $10.9 \mathrm{~km}$, con un tamaño de clastos líticos promedio de $3.5 \mathrm{~cm}$, mientras que el afloramiento más distal se encuentra a $19 \mathrm{~km}$ del Ajusco con un espesor de $1.56 \mathrm{~m}$ y dimensiones promedio de fragmentos líticos de $2.2 \mathrm{~cm}$ (Figura 6). El carácter masivo del depósito de pómez PBT sugiere que se depositó a partir de una columna eruptiva sostenida y estable y además con una distribución relativamente amplia, considerando la distancia entre los distintos afloramientos y sus espesores. Estas características sugieren que el depósito PBT fue producido por una erupción pliniana (Cioni et al., 2000). Adicionalmente, datos de parámetros sedimentológicos de la PBT comparados con datos de la PTS también se traslapan en cuanto a diámetro mediano de grano y grado de selección versus la distancia de la fuente (Figura 11).

\section{CONCLUSIONES}

El depósito denominado de manera informal como pómez Bosque de Tlalpan aflora en el suroeste de la cuenca de México con espesores máximos de $3 \mathrm{~m}$, constituido esencialmente por fragmentos de pómez del tamaño de lapilli grueso a bloques y escasos fragmentos líticos accidentales. La pómez es de color blanco, con fenocristales de plagioclasa, ortopiroxeno, anfíbol y biotita, así como escaso cuarzo y óxidos de Fe-Ti. La composición química de la pómez es dacítica, de afinidad calcialcalina y patrones de elementos traza y tierras raras característicos de magmatismo de arco, con las típicas anomalías negativas pronunciadas de $\mathrm{Nb}$ y $\mathrm{Ta}$. De acuerdo con los afloramientos descritos, el espesor y el tamaño de los fragmentos disminuye hacia el $\mathrm{NE}$, lo que sugiere que la fuente de emisión debería estar en sentido contrario, hacia el SW, en cuya región se encuentran los volcanes poligenéticos Ajusco y San Miguel. Con base en las isopacas preliminares y comparaciones químicas, se postula que la fuente de emisión fue el volcán Ajusco. Las edades de radiocarbono y termoluminscencia son muy similares, por lo que los paleosuelos fechados representan una edad máxima para este evento eruptivo que lo ubican en el Pleistoceno Tardío (entre 25,730 \pm 130 y 37,450 \pm 330 años A.P.). Además, el buen estado de preservación de la pómez en los afloramientos descritos
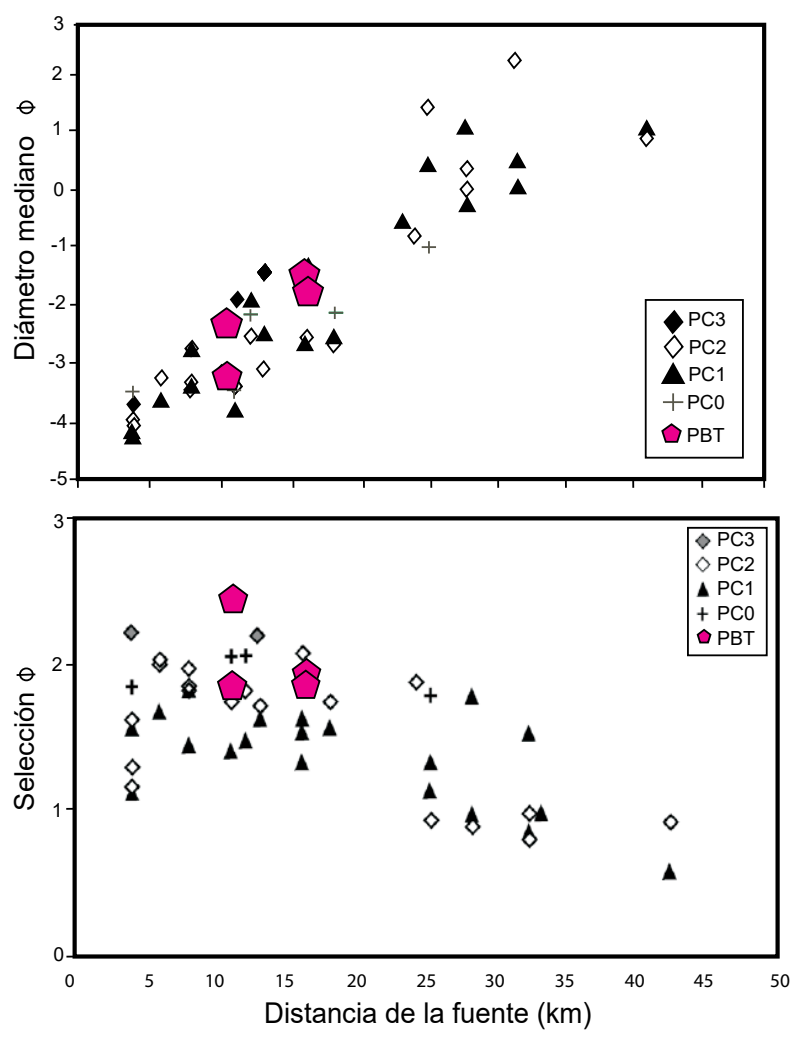

Figura 11. Diagramas de diámetro mediano y grado de selección vs. distancia de la fuente del depósito Pómez Toluca Superior, con clave PC0-PC3 (datos tomados de Arce et al., 2003), comparado con análisis sedimentológicos del depósito pómez Bosque de Tlalpan (PBT). Se aprecia que a una distancia de $10 \mathrm{~km}$, estos parámetros son similares para ambos depósitos.

también soporta la edad relativamente joven para este depósito. El depósito PBT fue producido por una erupción de tipo pliniano, dado el carácter masivo del depósito. Por lo tanto la PBT fue distribuida ampliamente en la cuenca de México y puede ser utilizada como un marcador estratigráfico en futuros estudios.

\section{AGRADECIMIENTOS}

Este estudio se llevó a cabo gracias al financiamiento del proyecto PAPIIT IN 104214 (J.L. Arce). Los análisis químicos de FRX se llevaron a cabo en el Laboratorio Nacional de Geoquímica y Mineralogía del Instituto de Geología, UNAM por la Quim. Patricia Girón García, y los análisis por ICP-MS se realizaron en el Laboratorio de Estudios Isotópicos (LEI) del Centro de Geociencias, UNAM por la M. en C. Ofelia Pérez Arvizu. Gracias a la M. en C. Elizabeth Rangel Granados por la revisión de la primera versión del manuscrito. Discusiones enriquecedoras con C. Siebe mejoraron las ideas planteadas en este artículo. Gracias a M.N. Guilbaud, L. Capra y un revisor anónimo por las críticas constructivas a este artículo, que sin duda mejoraron la versión final.

\section{REFERENCIAS}

Aguirre-Díaz, G.J., López-Martínez, M., Rendón-Márquez, G., 2006, La Caldera de La Catedral, Sierra de Las Cruces, una caldera al norponiente de la Ciudad de México, en Reunión Anual de la Unión Geofísica Mexicana: 
Puerto Vallarta, Jal., México, GEOS, 26, abstract, 160-161.

Aitken, M., 1985, Thermoluminescence Dating: Oxford, London, Academic Press, $370 \mathrm{pp}$.

Arce, J.L., Macías, J.L., Vázquez-Selem, L., 2003, The 10.5 ka Plinian eruption of Nevado de Toluca volcano, Mexico: Stratigraphy and hazard implications: Geological Society of America Bulletin, 115, 230-248.

Arce, J.L., Macías, R., García-Palomo, A., Capra, L., Macías, J.L., Layer, P., Rueda, H., 2008, Late Pleistocene flank collapse of Zempoala Volcano (Central Mexico) and the role of fault reactivation: Journal of Volcanology and Geothermal Research, 177, 944-958.

Arce, J.L., Layer, P.W., Morales-Casique, E., Benowitz, J.A., Rangel, E., Escolero, O., 2013a, New constraints on the subsurface geology of the Mexico City Basin: The San Lorenzo Tezonco deep well, on the basis of ${ }^{40} \mathrm{Ar}{ }^{39} \mathrm{Ar}$ geochronology and whole-rock chemistry: Journal of Volcanology and Geothermal Research, 266, 34-49.

Arce, J.L., Layer, P.W., Lassiter, J., Benowitz, J.A., Macías, J.L., RamírezEspinosa, J., 2013b, ${ }^{40} \mathrm{Ar} /{ }^{39} \mathrm{Ar}$ dating, geochemistry, and isotopic analyses of the Quaternary Chichinautzin Volcanic Field, south of Mexico City: Implications for timing, effusion rate, and distribution of the volcanism: Bulletin of Volcanology, 75:774, doi: 10.1007/s00445-013-0774-6.

Arce, J.L., Layer, P.W., Martínez, I., Salinas, J.I., Macías-Romo, M.C., MoralesCasique, E., Benowitz, J., Escolero, O., Lenhardt, N., 2015, Geología y estratigrafía del pozo profundo San Lorenzo Tezonco y de sus alrededores, sur de la Cuenca de México: Boletín de la Sociedad Geológica Mexicana, 67(2) 123-143.

Bloomfield, K., 1975, A late-Quaternary monogenetic volcano field in central Mexico: Geologische Rundschau, 64, 476-497.

Bloomfield, K., Sánchez-Rubio, G., Wilson, L., 1977, Plinian Eruptions of Nevado de Toluca: Geologische Rundschau, 66(1), 120-146.

Brown, E.T., Werne, J.P., Lozano-García, M.S., Caballero-Miranda, M., OrtegaGuerrero, B., Cabral-Cano, E., Valero-Garcés, B.L., Schwalb, A., ArciniegaCeballos, A., 2012, Workshop Reports, Scientific Drilling No. 14, 72-75. doi:10.2204/iodp.sd.14.12.2012.

Caballero, M.M., Ortega-Guerrero, B., 1998, Lake levels since about 40,000 years ago at Lake Chalco, near Mexico City: Quaternary Research, 50, 69-79.

Cadoux, A., Missenard, Y., Martínez-Serrano, R., Guillou, H., 2011, Trenchward Plio-Quaternary volcanism migration in the Trans-Mexican Volcanic Belt: the case of the Sierra Nevada range: Geological Magazine, doi:10.1017/ S0016756810000993.

Cioni, R., Marianelli, P., Santacroce, R., Sbrana, A., 2000, Plinian and Subplinian eruptions, en Sigurdsson, H. (ed.), Encyclopedia of Volcanoes: London, Academic Press, 477-494.

De Cserna, Z., De la Fuente-Duch, M., Palacios-Nieto, M., Triay, L., MitreSalazar, L.M., Mota-Palomino, R., 1988, Estructura geológica, gravimetría, sismicidad y relaciones neotectónicas regionales de la cuenca de México: Boletín del Instituto de Geología, Universidad Nacional Autónoma de México, 104, 1-71.

Fries, C., 1956, Bosquejo Geológico de la región entre México, D.F. y Taxco Guerrero, en Congreso Geológico Internacional 20: México, D.F., Libreto de las excursiones, Excursión, A-9 y C-12, 11.

Fries, C., 1960, Geología del Estado de Morelos y de partes adyacentes de México y Guerrero. Región central meridional de México: Boletín del Instituto de Geología, Universidad Nacional Autónoma de México, 60, 234 pp.

García-Palomo, A., Macías, J.L., Tolson, G., Valdez, G., Mora, J.C., 2008, Volcanic stratigraphy and geological evolution of the Apan región, eastcentral sector of the Trans-Mexican Volcanic Belt: Geofísica Internacional, 41, 133-150.

Guérin, G., Mercier, N., Adamiec, G., 2011, Dose-rate conversion factors: update: Ancient TL, 29-1, 5-8

Le Bas, M.J., Le Maitre, R.W., Streckaisen, A., Zanetti, B., 1986, A chemical classification of volcanic rocks based on the total alkali-silica diagram: Journal of Petrology, 27, 745-750.

Lenhardt, N., Böhnel, H., Wemmer, K., Torres-Alvarado, I.S., Hornung, J., Hinderer, M., 2010, Petrology, magnetostratigraphy and geochronology of the Miocene volcaniclastic Tepoztlán Formation: implications for the initiation of the Transmexican Volcanic Belt (Central Mexico): Bulletin of Volcanology, 72, 817-832.

Lozano-Barraza, L., 1968, Geología de la Sierra de Guadalupe: México, D.F., Instituto Politécnico Nacional, Escuela Superior de Ingeniería y
Arquitectura (ESIA), Tesis de Licenciatura, $43 \mathrm{pp}$.

Lozano-García, M.S., 1989, Palinología y paleoambientes pleistocénicos de la Cuenca de México: Geofísica Internacional, 28, 335-362.

Lozano-García, M.S., Ortega-Guerrero, B., 1998, Late Quaternary environmental changes of the central part of the Basin of Mexico: Correlation between Texcoco and Chalco basins: Review of Palaeobotany and Palynology, 99, 77-93.

Lozano-García, M.S., Ortega-Guerrero, B., Caballero-Miranda, M., UrrutiaFucugauchi, J., 1993, Late Pleistocene and Holocene paleoenvironments of Chalco Lake, central Mexico: Quaternary Research, 40, 332-342.

Lozano-Santacruz, R., Bernal, J.P., 2005, Characterization of a new set of eight geochemical reference materials for XRF major and trace element analysis: Revista Mexicana de Ciencias Geológicas, 22(3), 329-344.

Macias, J.L., Arce, J.L., García-Tenorio, F., Layer, P.W., Rueda, H., Reyes-Agustin, G., López-Pizaña, F., Avellán, D., 2012, Geology and geochronology of Tlaloc, Telapón, Iztaccíhuatl, and Popocatépetl volcanoes, Sierra Nevada, central Mexico, en Aranda-Gómez, J.J., Tolson, G., Molina-Garza, R.S. (eds.), The Southern Cordillera and Beyond: Geological Society of America Field Guide, 25,163-193.

Márquez, A., Oyarzu, R., Doblas, M., Verma, S.P., 1999, Alcalic (ocean-island basalt type) and calc-alkaline volcanism in the Mexican Volcanic Belt: A case for plume-related magmatism and propagating rifting at an active margin?: Geology, 27, 51-54.

Martin Del Pozzo, A.L., 1982, Monogenetic volcanism in Sierra Chichinautzin, Mexico: Bulletin of Volcanology, 45, 9-24.

Mejia, V., Böhnel, H., Opdyke, N.D., Ortega-Rivera, M.A., Lee, J.K.W., Aranda-Gómez, J.J., 2005, Paleosecular variation and time-averaged field recorded in late Pliocene-Holocene lava flows from Mexico: Geochemistry Geophysics Geosystems, 6, 1-19.

Mooser, F., 1963, Historia tectónica de la Cuenca de México: Boletin de la Asociación Mexicana de Geólogos Petroleros, 15, 239-245.

Mooser, F., 1975, Historia geológica de la Cuenca de México, en Memoria de las obras de drenaje profundo del Distrito Federal: México, D.F., Departamento del Distrito Federal, 1, 7-38.

Mora-Alvarez, G., Caballero, C., Urrutia-Fucugauchi, J., Uchiumi, S., 1991, Southward migration of volcanic activity in the Sierra de Las Cruces, Basin of Mexico. A preliminary K-Ar dating and paleomagnetic study: Geofísica Internacional, 30, 61-70.

Mori, L., Gómez-Tuena, A., Cai, Y., Goldstein, S.L., 2007, Effects of prolonged flat subduction on the Miocene magmatic record of the central TransMexican Volcanic Belt: Chemical Geology, 244, 452-473.

Nixon, G.T., 1989, The geology of Iztaccíhuatl volcano and adjacent areas of the Sierra Nevada and Valley of Mexico, en Nixon, G.T. (ed.) The geology of Iztaccíhuatl volcano and adjacent areas of the Sierra Nevada and Valley of Mexico: Geological Society of America Special Paper 219, 1-58.

Osete, M.L., Ruíz-Martínez, V.C., Caballero, M.C., Galindo, C., UrrutiaFucugauchi, J., Tarling, H.D., 2000, Southward migration of continental volcanic activity in the Sierra de Las Cruces, Mexico: Paleomagnetic and radiometric evidence: Tectonophysics, 318, 201-215.

PEMEX (Petróleos Mexicanos), 1987, Informe geológico de los pozos Mixhuca-1, Tulyehualco-1, Copilco-1 y Roma: Inédito.

Pérez-Cruz, G.A., 1988, Estudio sismológico de reflexión del subsuelo de la Ciudad de México: México D.F., Universidad Nacional Autónoma de México, Facultad de Ingeniería, Tesis de Maestría, 83 pp.

Pyle, D.M., 1989, The Thickness, volume and grainsize of tephra fall deposits Bulletin of Volcanology, 51, 1-15.

Rodríguez-Elizarrarás, S.R., Siebe, C., Komorowski, J.C., Abrams, M., 2002, The Quetzalapa Pumice: a voluminous late Pleistocene rhyolite deposit in the eastern Trans-Mexican Volcanic Belt: Journal of Volcanology and Geothermal Research, 113, 177-212.

Romero-Terán, E., 2001, Geología del Complejo Volcánico San Miguel, al sur poniente de la Cuenca de México: México D.F., Universidad Nacional Autónoma de México, Tesis de Maestía, 40 pp.

Saunders, A.D., Tarney, J., Weaver, S.D., 1980, Transversal geochemical variations across the Antarctic Peninsula: implication for the genesis of calc-alkaline magmas: Earth and Planetary Sciences Letters, 46, 344-360.

Siebe, C., 2000, Age and archaelogical implications of Xitle volcano, southwestern Basin of Mexico City: Journal of Volcanology and Geothermal Research, $104,45-64$ 
Siebe, C., Macías, J.L., 2004, Volcanic hazards in the Mexico City metropolitan area from eruptions at Popocatépetl, Nevado de Toluca, and Jocotitlán stratovolcanoes and monogenetic scoria cones in the Sierra Chichinautzin Volcanic Field, en Penrose Conference, Neogene-Quaternary Continental Margin Volcanism, State of Puebla, México, Field Guide: Boulder Colorado, Geological Society of America, 77 pp. doi: 10.1130/2004. VHITMC.PFG.

Siebe, C., Macias, J.L., Abrams, M., Rodriguez, S., Castro, R., Delgado, H., 1995, Quaternary explosive volcanism and pyroclastic deposits in east central mexico: implications for future hazards, en Chacko J. (ed.) Annual Meeting of the Geological Society of America, Field Trip Guidebook: Louisiana, United States, Geological Society of America, 1-48.

Sosa-Ceballos, G., Macías, J.L., García-Tenorio, F., Layer, P.W., Schaaf, P., Solís-Pichardo, G., Arce, J.L., 2015, El Ventorrillo, a paleostructure of Popocatépetl volcano: Insights from Geochronology and Geochemistry, 77, 1-20. doi:10.1007/s00445-015-0975-2.
Sun, S., McDonough, W., 1989, Chemical and isotopic systematics of oceanic basalts: implications for mantle compositions and processes, en Saunders, A., Norry, M., (eds.), Magmatism in ocean basins: Geological Society of London, Special Publication, 42, 313-345.

Vázquez-Sánchez, E., Jaimes-Palomera, R., 1989, Geología de la Cuenca de México: Geofísica Internacional, 28, 133-174

Manuscrito recibido: marzo 22, 2017

Manuscrito corregido recibido: octubre 17, 2017

Manuscrito aceptado: octubre 20, 2017 